\title{
OLIGONUCLEOTIDE LIGATION REACTIONS ON A CHIP USING MAGNETIC PARTICLES
}

\author{
Z. Hugh Fan; Rajan Kumar; Gregory Deffley; \\ Qianping Dong; Paul Stabile; Thomas Fare \\ Sarnoff Corporation, \\ CN 5300, Princeton, NJ 08543-5300 \\ E-mail: hfan@sarnoff.com
}

\begin{abstract}
We report the incorporation of magnetic particles into microfluidics for DNA diagnostics and discuss its advantages. Magnetic particles are transported using electroosmotic pumping to locations where they are held by a magnetic field. Annealing, ligation, and denaturation of oligonucleotides have been demonstrated on magnetic beads in microfabricated devices. The successful execution of these steps indicates the feasibility of isothermal nucleic acid amplification schemes using fluid cycling rather than thermal cycling.
\end{abstract}

\section{Introduction}

Fluid pumping used in most microfabricated devices results in the displacement of one solution with another at a particular location. As a result, chemical reactions have to be implemented along the channel flow, as demonstrated in microsystems using electroosmosis [1, 2]. Using this scheme, multi-step reactions such as DNA amplification are very difficult to execute unless the channel is unreasonably long. In addition, multiple side channels used to supply reagents will disturb the fluid flow system. To overcome these problems, we have incorporated magnetic particles into microfluidic systems. Sequential chemical reactions can take place on the surface of particles while the particles are held stationary by a magnetic field. The product of each step can be separated from undesired reagents simply by washing prior to the subsequent reaction. Fluid pumping does not displace the components on beads and multi-step reactions can be implemented at one location in a microfabricated device.

Nucleic acid amplification technologies, e.g., polymerase chain reaction (PCR) and ligase chain reaction (LCR), have been demonstrated in microfabricated devices [3-6]. Work to date has consisted of miniaturizing macroscale tubes $(\sim 1 \mathrm{ml})$ into microscale chambers $(\sim 10 \mu \mathrm{l})$, hence most features of microfluidics have not been fully utilized. We have conceived of isothermal amplification approaches that take advantage of microfluidics. Unlike thermal cycling in the conventional amplification methods, fluid cycling can be used to execute denaturation, annealing, and ligation (LCR) or extension (PCR). These three steps in each cycle are carried out by introducing appropriate reagents rather than adjusting temperatures. The advantages of this approach include isothermal reactions, fast analysis, and other benefits enabled by miniaturization [7]. To show the feasibility, we have demonstrated individual, roomtemperature reaction of each step, and the combination of oligonucleotide annealing and ligation reactions in a microfluidic system.

\section{Experimental Section}

Devices and Fabrication. Figure 1 shows the layout of the devices used for oligonucleotide ligation reactions. The channels are about $50 \mu \mathrm{m}$ wide and $20 \mu \mathrm{m}$ deep. The devices were made of two 54 × 54 × $0.5 \mathrm{~mm}$ glass plates. The channels were defined in one plate using photolithography. The holes at the channel ends were made in the second plate and they provide access to channels [8]. A glass plate with channels and a plate with holes were laminated together using a modified anodic bonding method described previously [9]. The device was then assembled with a Plexiglas fixture that is used for solution introductions and electrical connections.

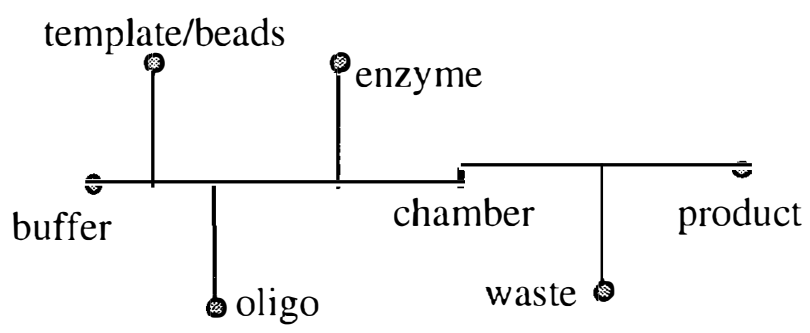

Figure 1. The lay'out of a device used for oligonucleotide ligation reactions.

Apparatus. An apparatus with voltage controls and a microscope system was assembled in-house. A Fluke high voltage power supply (Model 412B, Seattle, WA) was connected to a chip device via a control system. The system consists of relays and electronics that is able to simultaneously deliver ten different voltages for electroosmotic pumping. The voltages and the relays were controlled by a computer with a program written in $\mathrm{C}$ language. An inverted fluorescence microscope (Olympus IX70, Melville, NY) with a CCD color camera (Optronics, Goleta, CA) was used for monitoring transport of magnetic particles and fluorescence measurements. The images of 
beads and fluorescence were recorded on a VCR tape or acquired by an imaging software (MetaMorph, Universal Imaging, Hollis, NH).

Materials and Reagents. Corning 7740 (Pyrex) glass plates were purchased from Specialty Glass Products (Willow Grove, PA). Streptavidin-coated magnetic beads (Dynabeads ${ }^{\circledR}$ M-280) were procured from Dynal Inc. (Oslo, Norway) and T4 DNA ligase was from New England BioLabs (Beverly, MA). All oligonuleotides were obtained from Operon Technologies (Alameda, CA) and their sequences are listed in Table 1. The buffer used for ligation reactions consists of $50 \mathrm{mM}$ tris- $\mathrm{HCl}$ at $\mathrm{pH} 7.8,10 \mathrm{mM}$ $\mathrm{MgCl}_{2}, 10 \mathrm{mM}$ DTT, and $1 \mathrm{mM} \mathrm{ATP}$. A buffer solution of $\mathrm{pH} 8.2$ was prepared from $50 \mathrm{mM}$ boric acid and $50 \mathrm{mM}$ tris(hydroxymethyl) aminomethane (tris) (BioRad, Hercules, CA). All other chemicals are of reagent grade and all solutions were prepared in water from Milli-Qplus water system (Millipore, Bedford, MA). All solutions were filtered before use with a Millipore $0.22 \mu \mathrm{m}$ membrane filter unit.

Table 1. Sequences of oligonucleotides used in reactions.

\begin{tabular}{|c|l|}
\hline $\begin{array}{c}\text { LCRA } \\
\text { (24-mer) }\end{array}$ & $\begin{array}{l}\text { 5'-PTTGTGCCACGCGGTTGGGAATGT } \\
\text { A-3' }\end{array}$ \\
\hline LCRB & 5'-AGCAACGAATGTTTGCCCGCCAGT \\
(26-mer) & TG-3' \\
\hline $\begin{array}{c}\text { LCRTEMP } \\
\text { (60-mer) }\end{array}$ & $\begin{array}{l}\text { 5'-XCTGAATTACATTCCCAACCGCGT } \\
\text { GGCACAACAACTGGCGGGCAAACAG } \\
\text { TCGTTGCTGATT-3' }\end{array}$ \\
\hline mActin-4 & 5'-FTGTGGATCAGCAAGCAGGAGTAC \\
(30-mer) & GATGAGT-3'. \\
\hline mActin-8 & 5'-XACTCATCGTACTCCTGCTTGCTG \\
(30-mer) & ATCCACA-3'. \\
\hline
\end{tabular}

Note: $\mathrm{P}, \mathrm{X}$, and $\mathrm{F}$ stands for phosphate, biotin, and fluorescein groups, respectively.

Labeling and Gel Electrophoresis. The samples collected from the device were concentrated with seeDNA according to manufacturer's protocol (Amersham, Arlington Heights, IL). T4 kinase (New England BioLabs) and $\gamma-\mathrm{P}^{33}$ ATP (DuPont NEN, Boston, MA) were used to radioactively label oligonucleotides. Samples were then analyzed on a $12 \% \mathrm{C}$ polyacryamide slab gel. The gel electrophoresis was performed under denaturing conditions in $8 \mathrm{M}$ urea. The gel was dried and scanned using a phosphor imaging system (Storm 840, Molecular Dynamics, Sunnyvale, CA).

\section{Results and Discussions}

Bead Manipulation. Magnetic particles have been extensively used for the preparation, separation, and detection of biological molecules such as DNA, mostly because of their efficiency, simplicity, and low cost. We have applied biomagnetic techniques in microfabricated devices to perform sample preparation for DNA diagnostics, including capture of Escherichia coli using affinity beads and lysis of bacterial cells to extract DNA onto beads [10]. The transportation, localization, and manipulation of magnetic particles have been reproducibly performed in chips.

Electroosmotic pumping was exploited to transport magnetic particles and a permanent magnet was used to hold them. Figure 2 shows magnetic beads that were electroosmotically pumped to the chamber where the beads were captured by a magnet. Various types of particles have been examined using a range of pumping buffers. Dynabeads $^{\circledR}$ are very reliably pumped in $50 \mathrm{mM}$ tris-borate buffer at $\mathrm{pH} 8.2$.

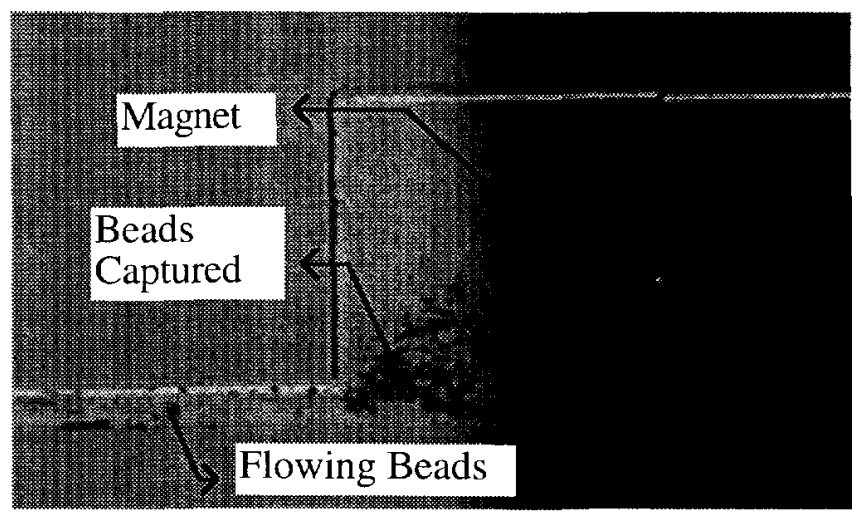

Figure 2. Dynabeads ${ }^{\bullet}(2.8 \mu \mathrm{m}$ diameter $)$ were electroosmotically pumped through a $50 \times 150 \mu \mathrm{m}$ channel into the chamber and then captured by a magnet. The chamber is $1 \mathrm{~mm}$ square with the depth of $50 \mu \mathrm{m} .500 \mathrm{~V}$ was applied to the channel, with an end-to-end distance of 38 $\mathrm{mm}$.

Annealing. Oligonucleotide probes need to hybridize with the template in order to be ligated by ligase. The annealing step was first demonstrated in a chip device by mixing beads with reagents (data not shown). Briefly, beads attached with a template (mActin-8) were introduced into the device using electroosmotic pumping and a magnet was used to hold the beads near the reaction chamber. After the accumulation of a certain number of beads, a fluorescentlylabelled probe (mActin-4) was pumped through these beads. The probe was expected to hybridize to the template because of their complementary sequences. After pumping a wash buffer through the beads, fluorescence on beads indicated the annealing of the probe on the template. The annealing between LCRTEMP and fluorescently labeled LCRA had also been confirmed in a chip before we pursued ligation reactions.

Ligation Reactions. Oligonucleotide ligation reactions were performed in the device shown in Figure 1. The reservoirs were filled with reagents as indicated. All steps involved in ligation reactions are schematically shown in Figure 3. Magnetic beads carrying a template (LCRTEMP) were pumped from the reservoir to the chamber by applying 
a voltage between the bead reservoir and the waste well. These beads were localized and accumulated in the chamber by a magnet. Two oligonucleotide probes (LCRA and LCRB) with complementary sequences to the template were then introduced into the chamber and hybridized with the template on beads. T4 DNA ligase in the ligase buffer was then pumped into the chamber and the enzyme ligated two hybridized probes. After the completion of the ligation, tris-borate buffer was pumped to wash non-specifically adsorbed oligonucleotides to waste. The magnet was then released and beads with ligated probes were transported to the product well by pumping the buffer again. The collected samples were denatured and radioactively labeled off-chip, followed by the analysis using gel electrophoresis. The gel electrophoregram in Figure 4 shows the presence of ligation products on the beads. The ligation yield is approximately $25 \%$ based on the intensities of oligo and ligated probe bands.

a. Template is attached to streptavidin-coated beads.

b. Two oligos anneal to the template.

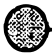

c. Ligase enables ligation of oligos.

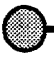

Figure 3. The protocol of oligonucleotide ligation reactions using DNA T4 ligase.

$$
\left.\left.\left|1^{1}\right|^{2}\right|^{3}\right|^{4} \mid
$$

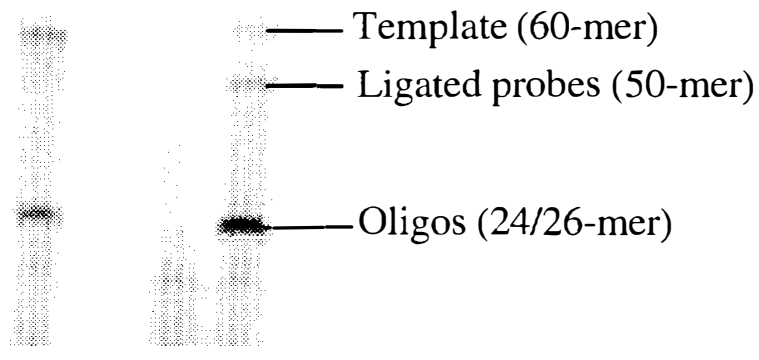

Figure 4. Gel electrophoregram showing ligation products on the beads. Lane I: standard (I fmol LCRA and I fmol LCRTEMP); Lane 2: empty lane; Lane 3: waste well; Lane 4: product well.
Denaturation. Denaturation of double-stranded DNA is the step to produce single-stranded DNA that can be used as a template for the hybridization of complementary oligonuleotide probes. The method used in conventional LCR (or PCR) is to heat the double-stranded DNA to a temperature near boiling. We employed $0.1 \mathrm{M} \mathrm{NaOH}$ rather than heating to denature DNA in the fluid cycling scheme. Electroosmotic pumping of $\mathrm{NaOH}$ was achieved by alternately transporting a plug of $\mathrm{NaOH}$ and a buffer [11]. Figure 5 shows the denaturation of an oligonucleotide probe (mActin-4) from a template (mActin-8) in a microfabricated device. The template was attached to beads via streptavidinbiotin conjugation. The fluorescently-labelled probe was annealed to the template in an Eppendorf tube. The beads with this double-stranded DNA were introduced into the device using electroosmotic pumping and a permanent magnet held the beads at a designated site. The top picture of Figure 5 shows a plug of fluorescent beads in the main channel of the device in Figure 1. After $\mathrm{NaOH}$ solution was electroosmotically pumped through the plug of beads, we observed the disappearance of fluorescence on beads, as shown in the bottom picture of Figure 5. This result indicates denaturation of DNA by $\mathrm{NaOH}$.
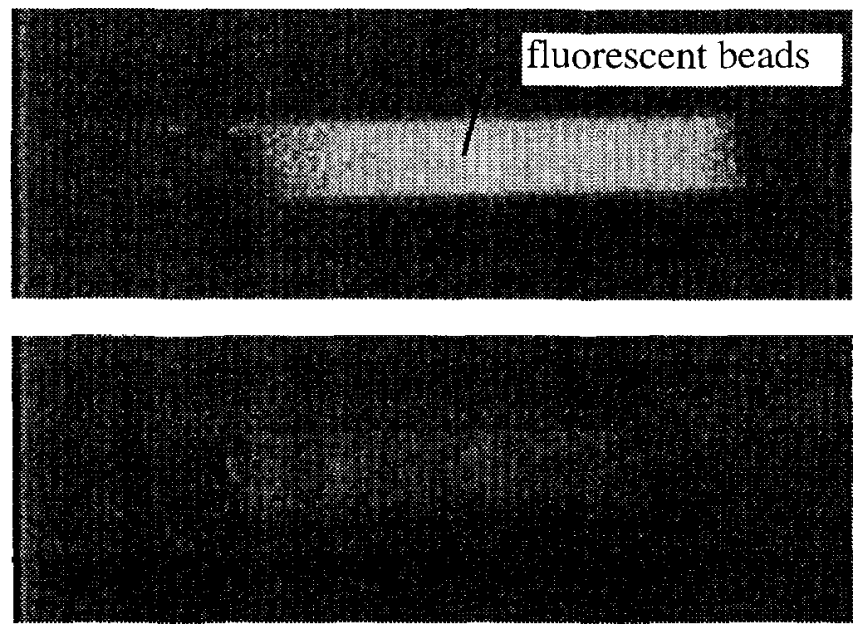

Figure 5. The room temperature denaturation of oligonucleotides using $0.1 \mathrm{M} \mathrm{NaOH}$ in a microfabricated device. (top) A plug of beads carnying a template annealed with a fluorescently-labelled oligonucleotide was electroosmotically pumped into the device and held in place by a magnet. (bottom) The same plug of beads shows little fluorescence after the denaturation step.

\section{Conclusion}

The advantages of using magnetic particles in microfluidic systems and heterogeneous chemical reactions between solid and liquid phases have been demonstrated. Many existing biomagnetic applications could be ransferred to microscale to take advantage of miniaturization [12]. The success of on-chip oligonucleotide annealing, ligation, 
and denaturation reactions indicates the feasibility of microfluidics-enabled amplification schemes. However, many challenges, such as the reaction yield, remain to be addressed.

Although radioactive labeling and gel electrophoresis were used as an analytical method to verify our approach, multi-probe hybridization will be the detection method of choice in the final device.

\section{References}

1. S. Hossein; T. Tang; D. J. Harrison, "Electroosmotic Pumping of Organic Solvents and Reagents in Microfabricated Reactor Chips", J. Am. Chem. Soc., 1997, 119, 8716-8717.

2. A. G. Hadd; D. E. Raymond; J. H. Halliwell; S. Jacobson; J. M. Ramsey, "Microchip Devices for Performing Enzyme Assays”, Anal. Chem., 1997, 69, 3407-3412.

3. M. A. Northrup; M. T. Ching; R. M. White; R. T. Watson, "DNA Amplification with a Microfabricated Reaction Chamber”, Transducers' 93, 1993, 924-926.

4. J. Cheng; M. A. Shoffner; K. R. Mitchelson; L. J. Kricka, P. Wilding; "Analysis of Ligase Chain Reaction Products Amplified in a Silicon-glass Chip Using Capillary Electrophoresis", J. Chromatogr. A, 1996, 732, 151-158.

5. A. T. Woolley; D. Hadley; P. Landre; A. J. deMello; R. A. Mathies; M. A. Northrup, "Functional Integration of PCR Amplification and Capillary Electrophoresis in a Microfabricated DNA Analysis Device", Anal. Chem., 1996, 68, 4081-4086.

6. L. C. Waters; S. C. Jacobson; N. Kroutchinina; J. Khandurina; R. S. Foote; J. M. Ramsey, "Microchip Devices for Cell lysis, Multiplex PCR Amplification, and Electrophoretic Sizing", Anal. Chem., 1998, 70, 158-162.

7. A. Manz; N. Graber; H. M. Widmer, "Miniaturized Total Chemcial Analysis Systems: a Novel Concept for Chemcial Sensing”, Sensors \& Actuators, 1990, Bl, 244-248.

8. Z. H. Fan; D. J. Harrison, "Micromachining of Capillary Electrophoresis Injectors and Separators on Glass Chips and Evaluation of Flow at Capillary Intersections", Anal. Chem., 1994, 66, 177-184.

9. Z. H. Fan; P. York; S. Cherukuri, "Chip Fabrication for Combinatorial Chemistry", Microstructures and Microfabricated Systems, Edited by P. J. Hesketh; G. Barna; H. G. Hughes, The Electrochemical Society, 1997, pp86-93.

10. Z. H. Fan; R. Kumar; P. Stabile, "On-Chip Cell Capture and Lysis Using Magnetic Particles", //th Internationational Symposium on High Performance Capillary Electrophoresis, Orlando, FL, Feb. 1-5, 1998, pp 109.
11. Z. H. Fan; T. Davis, G. Deffley, unpublished result.

12. L. G. Rashkovetsky; Y. V. Lyubarskaya; F. Foret; D. E. Hughes; B. L. Karger, "Automated Microanalysis Using Magnetic Beads with Commercial Capillary Electrophoretic Instrumentation", J. Chromatogr. A, 1997, 781, 197-204.

\section{Acknowledgment}

This work is financially supported by the Microflumes program managed by Dr. R. Ritts at the Defense Advanced Research Projects Agency (DARPA) and administered by SPAWAR Systems Center at San Diego, CA. Support from Drs. S. Cherukuri, P. York, and J. Ladd at Sarnoff is greatly appreciated. We acknowledge technical assistance and discussions from S. Perna, D. Ludington, B. Hoghooghi, D. Fishman, T. Davis, B. Lal, D. Lo Iacono, D. Pancholy, S. Lipp, and M. Massaro at Sarnoff. We are grateful for support from Orchid Biocomputer Inc., which is a Sarnoff technical venture responsible for commercializing this technology. 